\title{
Quantum copying: Beyond the no-cloning theorem
}

\author{
V. Bužek ${ }^{1,2}$ and M. Hillery ${ }^{1}$, \\ ${ }^{1}$ Department of Physics and Astronomy, Hunter College of the City University of New York, \\ 695 Park Avenue, New York, NY 10021, USA \\ 2 Institute of Physics, Slovak Academy of Sciences, Dúbravská cesta 9, 84228 Bratislava, Slovakia \\ (February 1, 2008) \\ We analyze a possibility of copying (三 cloning) of arbitrary states of quantum-mechanical spin- \\ $1 / 2$ system. We show that there exists a "universal quantum-copying machine" (i.e. transformation) \\ which approximately copies quantum-mechanical states such that the quality of its output does not \\ depend on the input. We also examine a machine which combines a unitary transformation and a \\ selective measurement to produce good copies of states in the neighborhood of a particular state. \\ We discuss the problem of measurement of the output states.
}

03.65. Bz 


\section{INTRODUCTION}

Suppose we have a quantum state $|s\rangle$ of a particular system which we would like to copy. For simplicity, assume that the state space of our system is two dimensional like that of a spin-1/2 particle, the polarizations of a photon, or a two level atom. We shall denote the basis elements by $|0\rangle_{a}$ and $|1\rangle_{a}$, where the subscript $a$ is used to indicate that this is the original system (which we shall often refer to as a mode with the polarization example in mind) which is to be copied. The state $|s\rangle_{a}$ is some linear combination of $|0\rangle_{a}$ and $|1\rangle_{a}$. We now want to feed $|s\rangle_{a}$ into a device, which we call a quantum copying machine, which at its output will give us $|s\rangle_{a}$ back and, in addition, a copy, i. e. a system identical to the one we put in which is also in the quantum state $|s\rangle$. Thus, we put in one system and get out two, both of which are in the same quantum state as the one which was fed into the input.

Let us now make this quantitative. The ideal copying process is described by the transformation

$$
|s\rangle_{a}|Q\rangle_{x} \longrightarrow|s\rangle_{a}|s\rangle_{b}|\tilde{Q}\rangle_{x}
$$

where $|s\rangle_{a}$ is the in-state of the original mode, $|Q\rangle_{x}$ is the $i n$-state of the copying device. The in-state of the copy mode $(b)$ is not specified in the transformation (1.1). In our discussion there is no need to specify this state, though in real physical processes this state can be assumed to be $|0\rangle_{b}$ (like a blank paper in a copying machine). The whole idea of quantum copying is to produce at the output of the copying machine two identical states $|s\rangle_{a}$ and $|s\rangle_{b}$ in the modes $a$ and $b$, respectively. The final state of the copying machine is described by the vector $|\tilde{Q}\rangle_{x}$.

First one has to ask a question: "Does quantum mechanics allow the transformation (1.1) for an arbitrary input state $|s\rangle_{a}$ ?". Wootters and Zurek [1] have answered this question. The answer is simple: "No". That is, quantum-mechanical states cannot be cloned (therefore the no-cloning theorem). To be more specific, the Wootters-Zurek no-cloning theorem tells us that quantum states cannot be cloned ideally for an arbitrary original in-state. This result has recently been extended to mixed states by Barnum, Caves, Fuchs, Jozsa, and Schumacher [2]. They have shown that if a particle in an arbitrary mixed state is sent into a device and two particles emerge, it is impossible for the two reduced density matrixes of the two-particle state to be identical to the input density matrix. Nevertheless, it is still an open question how well one can copy quantum states, i.e. when ideal copies are not available how close the copy state (outstate in the mode $b$ ) can be to the original state (i.e. $\left.|s\rangle_{a}\right)$. The other question to answer is what happens to the original state $|s\rangle_{a}$ after the copying. In the present paper we will illuminate these questions. In addition we will discuss how to measure the out-states after the copying procedure.
Why is quantum copying of interest? With the advent of quantum communication, e. g. quantum cryptography, and quantum computing, understanding the limits of the manipulations we can perform on quantum information becomes important. The no-cloning theorem is one such limit. It tells us that arbitrary quantum information cannot be copied exactly. On the other hand, we may be interested only in copying a restricted set of quantum bits, or qubits, approximately. Such a copy would allow us to gain some, but no all, information about the original. We would like to find out what we can do under these less restrictive conditions. We shall examine a number of possibilities. We shall first study a quantum copying machine of the type proposed by Wootters and Zurek in their proof of the no-cloning theorem. This machine has the property that the quality of the copy it makes depends on the input state. We shall next consider a copying machine for which this is not true, i. e. the quality of the copy is the same for all input states. We shall also look at a machine which is designed to copy well only a restricted set of input states. In particular, it copies states which are in the neighborhood of a particular state well, but copies states which are far from this state poorly. It would be reasonable to use a machine of this type if we are dealing with qubits which are near a given state. Finally we examine briefly the entanglement of the copy and the original. Because of this entanglement any measurement we perform on the copy will change the state of the original. We show how nonselective measurements can be used to minimize this problem.

The paper is organized as follows. In Section II we briefly describe the Wootters-Zurek copying procedure and we analyze quantum-statistical properties of the copied states. We introduce measures (Hilbert-Schmidt norms) on Hilbert space which allow us to quantify how "good" the copying procedure is. Section III is devoted to a description of the universal quantum copying which is input-state independent. That is we will describe a copying transformation for which the Hilbert-Schmidt norms under consideration are input-state independent. In Section IV we will analyze a measurement procedure by means of which one of the output states can be measured so the information about the second state can be obtained under the condition that it is least perturbed by the measurement procedure. In Section $\mathrm{V}$ we will discuss some specific quantum-copying transformations which produce very good copies in the neighborhood of specific input states. We finish the paper with conclusions.

\section{WOOTTERS-ZUREK QUANTUM COPYING MACHINE AND NON-CLONING THEOREM}

In their paper [1] Wootters and Zurek analyzed the copying process defined by the transformation relation 
on basis vectors $|0\rangle_{a}$ and $|1\rangle_{a}$ :

$$
\begin{gathered}
|0\rangle_{a}|Q\rangle_{x} \longrightarrow|0\rangle_{a}|0\rangle_{b}\left|Q_{0}\right\rangle_{x} \\
|1\rangle_{a}|Q\rangle_{x} \longrightarrow|1\rangle_{a}|1\rangle_{b}\left|Q_{1}\right\rangle_{x} .
\end{gathered}
$$

From the unitarity of the transformation process (2.1) and the orthonormality of the basis states $|0\rangle_{a}$ and $|1\rangle_{a}$ it follows that the copying-machine states $\left|Q_{0}\right\rangle_{x}$ and $\left|Q_{1}\right\rangle_{x}$ are normalized to unity, provided that ${ }_{x}\langle Q \mid Q\rangle_{x}=1$, i.e. we can assume that

$$
{ }_{x}\langle Q \mid Q\rangle_{x}={ }_{x}\left\langle Q_{0} \mid Q_{0}\right\rangle_{x}={ }_{x}\left\langle Q_{1} \mid Q_{1}\right\rangle_{x}=1 .
$$

The Wootters-Zurek (WZ) quantum copying machine (QCM) is defined in such a way that the basis vectors $|0\rangle_{a}$ and $|1\rangle_{a}$ are copied (三 cloned) ideally, that is, for these states the relation (1.1) is fulfilled. We note that the Wootters-Zurek copying machine is input-state dependent. Following Wootters and Zurek we check how the pure superposition state $|s\rangle_{a}$ (the so-called $S U(2)$ coherent state [3]) defined as

$$
|s\rangle_{a}=\alpha|0\rangle_{a}+\beta|1\rangle_{a}
$$

is copied by the copying machine described by the transformation relation (2.1). For simplicity we will assume in what follows that probability amplitudes $\alpha$ and $\beta$ are real and $\alpha^{2}+\beta^{2}=1$. Throughout the paper (except the Section 5) we consider $\alpha$ and $\beta$ to be real. Our results do not depend on this assumption and can be easily extended for complex $\alpha$ and $\beta$. Using the transformation relation (2.1) we obtain:

$$
|s\rangle_{a}|Q\rangle_{x} \longrightarrow \alpha|0\rangle_{a}|0\rangle_{b}\left|Q_{0}\right\rangle_{x}+\beta|1\rangle_{a}|1\rangle_{1}\left|Q_{1}\right\rangle_{x} \equiv|\Psi\rangle_{a b x}^{(o u t)} .
$$

If it is assumed that ${ }_{x}\left\langle Q_{0} \mid Q_{1}\right\rangle_{x}=0$, i.e. the two copyingmachine states $\left|Q_{0}\right\rangle_{x}$ and $\left|Q_{1}\right\rangle_{x}$ are orthonormal, then the reduced density operator $\hat{\rho}_{a b}^{(o u t)}$ describing the state of the original-copy subsystem after the copying procedure reads:

$$
\hat{\rho}_{a b}^{(\text {out })}=\operatorname{Tr}_{x}\left[\hat{\rho}_{a b x}^{(\text {out })}\right]=\alpha^{2}|00\rangle\left\langle 00\left|+\beta^{2}\right| 11\right\rangle\langle 11|,
$$

where $\hat{\rho}_{a b x}^{(o u t)} \equiv|\Psi\rangle_{a b x}^{(o u t)}{ }_{a b x}^{(o u t)}\langle\Psi|$ [see Eq.(2.4)] and the basis vectors associated with two two-level systems under consideration are defined as

$$
\begin{gathered}
|00\rangle \equiv|0\rangle_{a}|0\rangle_{b} ; \quad|11\rangle \equiv|1\rangle_{a}|1\rangle_{b} \\
|01\rangle \equiv|0\rangle_{a}|1\rangle_{b} ; \quad|10\rangle \equiv|1\rangle_{a}|0\rangle_{b} .
\end{gathered}
$$

We also introduce two vectors $| \pm\rangle$

$$
|+\rangle=\frac{1}{\sqrt{2}}(|10\rangle+|01\rangle) ; \quad|-\rangle=\frac{1}{\sqrt{2}}(|10\rangle-|01\rangle) .
$$

which together with $|00\rangle$ and $|11\rangle$ create an orthonormal basis. Density operators describing quantum states of the original mode and the copy mode after the copying procedure read

$$
\begin{aligned}
& \hat{\rho}_{a}^{(\text {out })}=\operatorname{Tr}_{b}\left[\hat{\rho}_{a b}^{\text {(out })}\right]=\alpha^{2}|0\rangle_{a a}\left\langle 0\left|+\beta^{2}\right| 1\right\rangle_{a a}\langle 1|, \\
& \hat{\rho}_{b}^{(\text {out })}=\operatorname{Tr}_{a}\left[\hat{\rho}_{a b}^{(\text {out })}\right]=\alpha^{2}|0\rangle_{b b}\left\langle 0\left|+\beta^{2}\right| 1\right\rangle_{b b}\langle 1|,
\end{aligned}
$$

respectively. From Eq.(2.7) it follows that both the original and the copy mode at the output are in identical states (this is a good news), but the original mode at the output is in a mixture state (all off-diagonal elements are destroyed).

In order to judge how good the copying machine is we need a way of comparing its output to what, ideally, its output should be. That is, we need a way of comparing density matrixes. We shall use the square of the Hilbert-Schmidt norm of the difference between two density matrixes as a measure of how close they are to each other. The Hilbert-Schmidt norm of an operator, $\hat{A}$, is given by

$$
\|\hat{A}\|_{2}=\left[\operatorname{Tr}\left(\hat{A}^{\dagger} \hat{A}\right)\right]^{1 / 2},
$$

and it has the property that for operators $\hat{A}$ and $\hat{B}$

$$
\left|\operatorname{Tr}\left(\hat{A}^{\dagger} \hat{B}\right)\right| \leq\|\hat{A}\|_{2}\|\hat{B}\|_{2} .
$$

Our distance between the density matrixes $\hat{\rho}_{1}$ and $\hat{\rho}_{2}$ is then

$$
D=\left(\left\|\hat{\rho}_{1}-\hat{\rho}_{2}\right\|_{2}\right)^{2} .
$$

Is this a reasonable measure? In a two-dimensional space any observable, $A$, which is not a multiple of the identity, is represented by an hermitian operator, $\hat{A}$, which can be expressed as

$$
\hat{A}=\lambda_{1} \hat{P}_{1}+\lambda_{2} \hat{P}_{2},
$$

where $\lambda_{1}$ and $\lambda_{2}$ are real and $\hat{P}_{1}$ and $\hat{P}_{2}$ are hermitian, one-dimensional projections with the property that $\hat{P}_{1} \hat{P}_{2}=0$. For a given density matrix, $\hat{\rho}$, the probability that $A$ takes the value $\lambda_{i}$ is given by

$$
p_{i}=\operatorname{Tr}\left(\hat{\rho} \hat{P}_{i}\right) .
$$

We would like our notion of closeness for density matrixes to have the property that if two density matrixes are close, then the probability distributions generated by them for an arbitrary observable $A$ are also close. That is, if $\hat{\rho}_{1}$ and $\hat{\rho}_{2}$ are close, then the probability that $A$ takes the value $\lambda_{i}$ in the state $\hat{\rho}_{1}, p_{i}^{(1)}$, should be close to the probability that $A$ takes the value $\lambda_{i}$ in the state $\hat{\rho}_{2}$, $p_{i}^{(2)}$. Using the property of the Hilbert-Schmidt norm given in Eq. (2.9), we can, in fact, show that if the 
Hilbert-Schmidt norm of $\left(\hat{\rho}_{1}-\hat{\rho}_{2}\right)$ is small, then $p_{i}^{(1)}$ will be close to $p_{i}^{(2)}$. We have

$$
\begin{aligned}
& \left|p_{i}^{(1)}-p_{i}^{(2)}\right|=\left|\operatorname{Tr}\left[\hat{P}_{i}\left(\hat{\rho}_{1}-\hat{\rho}_{2}\right)\right]\right| \\
& \leq\left\|\hat{P}_{i}\right\|_{2}\left\|\hat{\rho}_{1}-\hat{\rho}_{2}\right\|_{2}=\left\|\hat{\rho}_{1}-\hat{\rho}_{2}\right\|_{2},
\end{aligned}
$$

where we have used the fact that the Hilbert-Schmidt norm of a one-dimensional projection is one. This shows that our proposed definition of closeness based on the Hilbert-Schmidt norm has the desired property and allows us to maintain that, in a two-dimensional space, it is a reasonable definition to use.

Other measures of the similarity of two density matrixes have been used. Schumacher [4] has advocated the use of fidelity which is defined as

$$
F=\operatorname{Tr}\left(\hat{\rho}_{1}^{1 / 2} \hat{\rho}_{2} \hat{\rho}_{1}^{1 / 2}\right)^{1 / 2},
$$

which ranges between 0 and 1 . A fidelity of one means two density matrixes are equal. This is a more satisfactory definition in general. The interpretation of the Hilbert-Schmidt norm in terms of probability distributions breaks down in infinite dimensional spaces and becomes less good in finite dimensional spaces as the dimension increases. Hilbert-Schmidt norms are, on the other hand, easier to calculate than fidelities. For our purposes, in a two-dimensional space, the Hilbert-Schmidt norm provides a very reasonable way to compare density matrixes.

To see how "far" the copying machine drives the original mode from its initial state we now evaluate the Hilbert-Schmidt norm, i.e. the "distance" between the in- and out-density operators of the original mode. The Hilbert-Schmidt norm is defined as

$$
D_{a} \equiv \operatorname{Tr}\left[\hat{\rho}_{a}^{(i d)}-\hat{\rho}_{a}^{(\text {out })}\right]^{2},
$$

where we denote the input density operator of the original mode as $\hat{\rho}_{a}^{(i d)}$ (here index id stands for the ideal). This density operator of the state (2.3) reads

$$
\hat{\rho}_{a}^{(i d)}=\alpha^{2}|0\rangle_{a}\langle 0|+\alpha \beta| 0\rangle_{a}\langle 1|+\beta \alpha| 1\rangle_{a}\left\langle 0\left|+\beta^{2}\right| 1\right\rangle_{a}\langle 1| .
$$

The Hilbert-Schmidt norm of the difference between the density operators (2.7a) and (2.16) is

$$
D_{a}=2 \alpha^{2} \beta^{2}=2 \alpha^{2}\left(1-\alpha^{2}\right),
$$

which clearly reflects the fact that the states $|0\rangle_{a}$ (i.e. $\alpha=1$ ) and $|1\rangle_{a}$ (i.e. $\alpha=0$ ) are copied perfectly, that is for these states $D_{a}=0$, while the pure superposition states $|s\rangle_{a}=\left(|0\rangle_{a} \pm|1\rangle_{a}\right) / \sqrt{2}$ are copied worst. In this case $D_{a}=1 / 2$. We remind ourselves that the maximum possible value for the Hilbert-Schmidt norm of the difference of two density matrixes is equal to two (for instance, this is the "distance" between two mutually orthogonal states $|0\rangle_{a}$ and $|1\rangle_{a}$ ). If we do not specify which pure superposition state $|s\rangle_{a}$ is going to be copied (i.e. we do not know a priori the value of $\alpha$ ) then on average we should expect the distance $D_{a}$ in the case of the Wootters-Zurek copying machine to be

$$
\bar{D}_{a}=\int_{0}^{1} d \alpha^{2} D_{a}\left(\alpha^{2}\right)=\frac{1}{3}
$$

From Eq.(2.17) we see that the Wootters-Zurek quantum copying procedure is state-dependent, that is for some states it operates well (even perfectly) while for some states it operates badly. Moreover, as it follows from Eq.(2.5) the output modes are, in general, highly entangled, which is not what we would expect from a perfect copying machine for which the output density operator $\hat{\rho}_{a b}^{(i d)}$ should be expressed as

$$
\hat{\rho}_{a b}^{(i d)}=\hat{\rho}_{a}^{(i d)} \otimes \hat{\rho}_{b}^{(i d)},
$$

where the density operators of the ideal original and the copy at the output are described by Eq.(2.16). The density operator $\hat{\rho}_{a b}^{(i d)}$ in the basis (2.6) reads

$$
\begin{aligned}
& \hat{\rho}_{a b}^{(i d)}=\alpha^{4}|00\rangle\left\langle 00\left|+\sqrt{2} \alpha^{3} \beta\right| 00\right\rangle\left\langle+\left|+\alpha^{2} \beta^{2}\right| 00\right\rangle\langle 11| \\
& +\sqrt{2} \alpha^{3} \beta|+\rangle\left\langle 00\left|+2 \alpha^{2} \beta^{2}\right|+\right\rangle\left\langle+\left|+\sqrt{2} \alpha \beta^{3}\right|+\right\rangle\langle 11| \\
& +\alpha^{2} \beta^{2}|11\rangle\left\langle 00\left|+\sqrt{2} \alpha \beta^{3}\right| 11\right\rangle\left\langle+\left|+\beta^{4}\right| 11\right\rangle\langle 11| .
\end{aligned}
$$

To measure the degree of entanglement we can either use the entropic parameter $S_{a b}$ as proposed by Barnett and Phoenix [5], or we can use the Hilbert-Schmidt norm $D_{a b}$ measuring the "distance" between the actual two-mode density operator $\hat{\rho}_{a b}^{(\text {out })}$ and a direct product of density operators $\hat{\rho}_{a}^{(\text {out })}$ and $\hat{\rho}_{b}^{(\text {out })}$, i.e.

$$
D_{a b}^{(1)}=\operatorname{Tr}\left[\hat{\rho}_{a b}^{(\text {out })}-\hat{\rho}_{a}^{(\text {out })} \otimes \hat{\rho}_{b}^{(\text {out })}\right]^{2} .
$$

Using the explicit expressions for the density operators which appear in Eq.(2.20) we find the Hilbert-Schmidt norm to be

$$
D_{a b}^{(1)}=D_{a} D_{b},
$$

where the single-mode norms $D_{a}\left(D_{b}\right)$ are given by Eq.(2.17). Analogously we can evaluate the HilbertSchmidt norm for the density operators $\hat{\rho}_{a b}^{(o u t)}$ and $\hat{\rho}_{a b}^{(i d)}$ [see Eqs.(2.5) and (2.19), respectively]. In this case we find

$$
D_{a b}^{(2)}=\operatorname{Tr}\left[\hat{\rho}_{a b}^{(o u t)}-\hat{\rho}_{a b}^{(i d)}\right]^{2}=D_{a}+D_{b} .
$$

Note that this result and Eq. (2.21) imply that the output state is most entangled when the performance of the 
copying machine is worst. To complete the picture we evaluate the distance $D_{a b}^{(3)}$ between the ideal output described by the density operator $\hat{\rho}_{a b}^{(i d)}$ and the direct product of the single-mode density operators

$$
D_{a b}^{(3)}=\operatorname{Tr}\left[\hat{\rho}_{a b}^{(i d)}-\hat{\rho}_{a}^{(\text {out })} \otimes \hat{\rho}_{b}^{(\text {out })}\right]^{2}=D_{a}+D_{b}-D_{a b}^{(1)} .
$$

From the above equations we clearly see that the output modes are firstly, entangled (except the cases when $\alpha^{2}=0$ or $\alpha^{2}=1$ ). Secondly, the degree of entanglement quantified via the norm $D_{a b}^{(1)}$ is initial-state dependent (i.e. it depends on the parameter $\alpha^{2}$ ). Thirdly, there is the following relationship between the norms $D_{a b}^{(i)}$

$$
D_{a b}^{(1)} \leq D_{a b}^{(3)} \leq D_{a b}^{(2)} .
$$

For further reference we note that the input-state averaged value [see equations Eq.(2.18) and (2.22)] of the norm $\bar{D}_{a b}^{(2)}$ is equal to $2 / 3$, while the input-averaged values of $\bar{D}_{a b}^{(1)}$ and $\bar{D}_{a b}^{(3)}$ are $2 / 15$ and $8 / 15$, respectively.

We finish the present section on the Wootters-Zurek quantum copying procedure with several comments.

(1) If we assume the original $(a)$ mode to be initially in the mixture state

$$
\hat{\rho}_{a}^{(i n)}=\alpha^{2}|0\rangle_{a a}\left\langle 0\left|+\beta^{2}\right| 1\right\rangle_{a_{a}}\langle 1|,
$$

then the output density operator describing the modes $a$ and $b$ after the copying is given by the same relation (2.5) as in the case of the pure input state. This means that if the input $a$ mode is described by the density operator (2.25), then the input and the output density operators in the mode $a$ are equal, i.e. $D_{a}=0$. Nevertheless, the distance $D_{a b}^{(1)}$ reflecting a degree of entanglement between the output modes has the value equal to $4 \alpha^{4} \beta^{4}$, i.e. is the same as for the pure input state (2.3). This simply reflects the fact, that the Wootters-Zurek quantum copying machine produces a strong entanglement between output modes even in the case when "classical" states (mixtures) are copied.

(2) It is interesting to note that the Wootters-Zurek QCM preserves the initial mean value of the operator $\hat{\sigma}_{z}=(|1\rangle\langle 1|-| 0\rangle\langle 0|) / 2$ while it completely destroys any information about the initial mean value of the operator $\hat{\sigma}_{x}=(|1\rangle\langle 0|+| 0\rangle\langle 1|) / 2$, that is

$$
\left\langle\hat{\sigma}_{z}\right\rangle_{a}^{(i n)}=\left\langle\hat{\sigma}_{z}\right\rangle_{a}^{(o u t)}=-\frac{1}{2} \cos 2 \phi,
$$

where we have used the parameterization $\alpha=\cos \phi$ and $\beta=\sin \phi$. On the other hand

$$
\left\langle\hat{\sigma}_{x}\right\rangle_{a}^{(i n)}=\frac{1}{2} \sin 2 \phi,
$$

while $\left\langle\hat{\sigma}_{x}\right\rangle_{a}^{(\text {out })}=0$ irrespective of the initial state of the original mode $a$. This means that whatever the value of the initial variance $\left\langle\left(\Delta \hat{\sigma}_{x}\right)^{2}\right\rangle_{a}^{(i n)}$ its output value is equal to $1 / 4$. These observations suggest that the WoottersZurek QCM is "designed" in such a way that the mean value of the operator $\left(\hat{\sigma}_{z}\right)$ is preserved by the copying procedure, while information associated with other mean values is totally destroyed. This in turn suggests that one can think about designing a copying machine associated with certain observation levels [6].

(3) Finally we briefly note that the von Neumann entropy [7] can also be utilized to describe the "quality" of the original and copy modes. The von Neumann entropy of a quantum-mechanical system described by the density operator $\hat{\rho}$ is defined as

$$
S=-k_{\mathrm{B}} \operatorname{Tr}[\hat{\rho} \ln \hat{\rho}] .
$$

Due to the Araki-Lieb theorem [8] and the fact that we consider a conservative system for which the entropy of the complete system is constant, and is equal to zero providing both modes $a$ and $b$ are initially in pure states, the entropy of the QCM $\left(S_{x}\right)$ after the copying is equal to the entropy $\left(S_{a b}\right)$ of the original-copy subsystem described by the density operator $\hat{\rho}_{a b}^{(o u t)}$. Besides this general property we find that

$$
S_{a b}=S_{a}=S_{b}=-k_{\mathrm{B}}\left[\alpha^{2} \ln \alpha^{2}+\beta^{2} \ln \beta^{2}\right],
$$

irrespective of whether the original mode has been initially prepared in the pure state (2.3) or a corresponding statistical mixture described by the density operator (2.25). If the output state were a product of states in the $a$ and $b$ modes we would have $S_{a b}=S_{a}+S_{b}$. The fact that the entropy is smaller than this shows that the modes are correlated, i. e. entangled.

\section{INPUT-STATE-INDEPENDENT QUANTUM COPYING MACHINE}

The Wootters-Zurek QCM suffers one significant disadvantage - its operation depends on the state of the original input. That is, the states $|0\rangle$ and $|1\rangle$ are copied perfectly, but the superposition states $(|0\rangle \pm|1\rangle) / \sqrt{2}$ are essentially destroyed by this particular copying machine in the sense that information about quantum coherences (off-diagonal elements of the density operator in a considered basis) is eliminated.

In what follows we describe a copying process which is input-state independent. When using this "universal" quantum copying machine (UQCM) superposition states (2.3) are copied equally well for any value of $\alpha$ in a sense that the distances $D_{a}=\operatorname{Tr}\left[\hat{\rho}_{a}^{(o u t)}-\hat{\rho}_{a}^{(i d)}\right]^{2}$ and $D_{a b}=$ $\operatorname{Tr}\left[\hat{\rho}_{a b}^{(o u t)}-\hat{\rho}_{a b}^{(i d)}\right]^{2}$ do not depend on the parameter $\alpha$. In addition to this we design the UQCM in such way that both $D_{a}$ and $D_{a b}$ take minimal values.

The most general quantum-copying transformation rules for pure states on a two-dimensional space can be 
written as

$$
\begin{array}{r}
|0\rangle_{a}|Q\rangle_{x} \longrightarrow \sum_{k, l=0}^{1}|k\rangle_{a}|l\rangle_{b}\left|Q_{k l}\right\rangle_{x} \\
|1\rangle_{a}|Q\rangle_{x} \longrightarrow \sum_{m, n=0}^{1}|m\rangle_{a}|n\rangle_{b}\left|Q_{m n}\right\rangle_{x}
\end{array}
$$

where the states $\left|Q_{m n}\right\rangle_{x}$ are not necessarily orthonormal for all possible values of $m$ and $n$. The general copying transformation is very complex and it involves many free parameters ${ }_{x}\left\langle Q_{k l} \mid Q_{m n}\right\rangle_{x}$ which characterize the copying machine. In what follows we will concentrate our attention on one particular copying-transformation which fulfills our demands as described above. We propose the following transformation

$$
\begin{array}{r}
|0\rangle_{a}|Q\rangle_{x} \longrightarrow|0\rangle_{a}|0\rangle_{b}|Q 0\rangle_{x}+\left[|0\rangle_{a}|1\rangle_{b}+|1\rangle_{a}|0\rangle_{b}\right]\left|Y_{0}\right\rangle_{x} \\
3.2 a \\
|1\rangle_{a}|Q\rangle_{x} \longrightarrow|1\rangle_{a}|1\rangle_{b}\left|Q_{1}\right\rangle_{x}+\left[|0\rangle_{a}|1\rangle_{b}+|1\rangle_{a}|0\rangle_{b}\right]\left|Y_{1}\right\rangle_{x}
\end{array}
$$

which is an obvious generalization of the WZ QCM. Due to the unitarity of the transformation (3.2) the following relations hold

$$
\begin{gathered}
{ }_{x}\left\langle Q_{i} \mid Q_{i}\right\rangle_{x}+2{ }_{x}\left\langle Y_{i} \mid Y_{i}\right\rangle_{x}=1 ; \quad i=0,1 \\
{ }_{x}\left\langle Y_{0} \mid Y_{1}\right\rangle_{x}={ }_{x}\left\langle Y_{1} \mid Y_{0}\right\rangle_{x}=0 .
\end{gathered}
$$

There are still many free parameters to specify, therefore we will further assume that the copying-machine state vectors $\left|Y_{i}\right\rangle_{x}$ and $\left|Q_{i}\right\rangle_{x}$ are mutually orthogonal:

$$
{ }_{x}\left\langle Q_{i} \mid Y_{i}\right\rangle_{x}=0 ; \quad i=0,1,
$$

and that

$$
{ }_{x}\left\langle Q_{0} \mid Q_{1}\right\rangle_{x}=0
$$

With these assumptions in mind we find the density operator $\hat{\rho}_{a b}^{(o u t)}$ describing the modes $a$ and $b$ after copying of the pure superposition state (2.3) as:

$$
\begin{gathered}
\hat{\rho}_{a b}^{(\text {out })}=\alpha^{2}|00\rangle\left\langle\left. 00\right|_{x}\left\langle Q_{0} \mid Q_{0}\right\rangle_{x}+\sqrt{2} \alpha \beta \mid 00\right\rangle\left\langle+{ }_{x}\left\langle Y_{1} \mid Q_{0}\right\rangle_{x}\right. \\
+\sqrt{2} \alpha \beta|+\rangle\left\langle\left. 00\right|_{x}\left\langle Q_{0} \mid Y_{1}\right\rangle_{x}+\left[2 \alpha^{2}{ }_{x}\left\langle Y_{0} \mid Y_{0}\right\rangle_{x}\right.\right. \\
\left.+2 \beta^{2}{ }_{x}\left\langle Y_{1} \mid Y_{1}\right\rangle_{x}\right]|+\rangle\langle+|+\sqrt{2} \alpha \beta|+\rangle\left\langle\left. 11\right|_{x}\left\langle Q_{1} \mid Y_{0}\right\rangle_{x} \quad(3.5)\right. \\
+\sqrt{2} \alpha \beta|11\rangle\left\langle+\left|{ }_{x}\left\langle Y_{0} \mid Q_{1}\right\rangle_{x}+\beta^{2}\right| 11\right\rangle\left\langle\left. 11\right|_{x}\left\langle Q_{1} \mid Q_{1}\right\rangle_{x} .\right.
\end{gathered}
$$

The density operator describing the $a$ mode can be obtained from Eq.(3.5) by tracing over the mode $b$ and it reads

$$
\hat{\rho}_{a}^{(\text {out })}=|0\rangle_{a a}\langle 0|\left[\alpha^{2}+\left(\beta^{2}{ }_{x}\left\langle Y_{1} \mid Y_{1}\right\rangle_{x}-\alpha_{x}^{2}\left\langle Y_{0} \mid Y_{0}\right\rangle_{x}\right)\right]
$$

$$
\begin{gathered}
+|0\rangle_{a}{ }_{a}\langle 1| \alpha \beta\left[{ }_{x}\left\langle Q_{1} \mid Y_{0}\right\rangle_{x}+{ }_{x}\left\langle Y_{1} \mid Q_{0}\right\rangle_{x}\right] \\
+|1\rangle_{a}{ }_{a}\langle 0| \alpha \beta\left[{ }_{x}\left\langle Q_{0} \mid Y_{1}\right\rangle_{x}+{ }_{x}\left\langle Y_{0} \mid Q_{1}\right\rangle_{x}\right] \\
+|1\rangle_{a}{ }_{a}\langle 1|\left[\beta^{2}+\left(\alpha^{2}{ }_{x}\left\langle Y_{0} \mid Y_{0}\right\rangle_{x}-\beta^{2}{ }_{x}\left\langle Y_{1} \mid Y_{1}\right\rangle_{x}\right)\right] .
\end{gathered}
$$

The density operator $\hat{\rho}_{b}^{(\text {out })}$ looks exactly the same. This means that the states of the two modes $a$ and $b$ at the output of the copying machine under consideration are equal to each other, but they are not equal to the density operator of the in-state of the original mode [compare Eqs.(3.6) and (2.16)]. This means that the original state is distorted by the copying. To quantify the degree of this distortion we evaluate the Hilbert-Schmidt norm (2.10) for the density operators (3.6) and (2.16):

$$
D_{a}=2 \xi^{2}\left(4 \alpha^{4}-4 \alpha^{2}+1\right)+2 \alpha^{2}\left(1-\alpha^{2}\right)(\eta-1)^{2},
$$

where we have introduced the notation

$$
{ }_{x}\left\langle Y_{0} \mid Y_{0}\right\rangle_{x}={ }_{x}\left\langle Y_{1} \mid Y_{1}\right\rangle_{x} \equiv \xi
$$

${ }_{x}\left\langle Y_{0} \mid Q_{1}\right\rangle_{x}={ }_{x}\left\langle Q_{0} \mid Y_{1}\right\rangle_{x}={ }_{x}\left\langle Q_{1} \mid Y_{0}\right\rangle_{x}={ }_{x}\left\langle Y_{1} \mid Q_{0}\right\rangle_{x} \equiv \eta / 2$,

with $0 \leq \xi \leq 1 / 2$ and $0 \leq \eta \leq 2 \xi^{1 / 2}(1-2 \xi)^{1 / 2} \leq$ $1 / \sqrt{2}$, which follows from the Schwarz inequality. The relation (3.8) further specifies "properties" of the copying machine under consideration. So essentially we end up with two "free" parameters which we will specify further.

As we said in the introduction, we are looking for a copying machine such that all input original state are copied equally well, that is we want the norm (3.7) to be independent of the parameter $\alpha^{2}$. This means that one of the parameters $\xi$ or $\eta$ can be determined from the condition

$$
\frac{\partial}{\partial \alpha^{2}} D_{a}=0
$$

where the norm $D_{a}$ is given by Eq.(3.7). From Eq.(3.9) we find that if the parameters $\xi$ and $\eta$ are related as

$$
\eta=1-2 \xi
$$

then the norm $D_{a}$ is input-state independent and it takes the value

$$
D_{a}=2 \xi^{2}
$$

Taking into account the relations (3.10) and (3.3a) we can rewrite now the density operators $\hat{\rho}_{a b}^{(o u t)}$ and $\hat{\rho}_{a}^{(o u t)}$ [see Eqs.(3.5) and (3.6), respectively] as

$$
\begin{gathered}
\hat{\rho}_{a b}^{\text {(out })}=\alpha^{2}(1-2 \xi)|00\rangle\left\langle 00\left|+\frac{\alpha \beta}{\sqrt{2}}(1-2 \xi)\right| 00\right\rangle\langle+| \\
+\frac{\alpha \beta}{\sqrt{2}}(1-2 \xi)|+\rangle\langle 00|+2 \xi|+\rangle\left\langle+\left|+\frac{\alpha \beta}{\sqrt{2}}(1-2 \xi)\right|+\right\rangle\langle 11|
\end{gathered}
$$




$$
+\frac{\alpha \beta}{\sqrt{2}}(1-2 \xi)|11\rangle\left\langle+\left|+\beta^{2}(1-2 \xi)\right| 11\right\rangle\langle 11|
$$

and

$$
\begin{aligned}
& \hat{\rho}_{a}^{(\text {out })}=|0\rangle_{a_{a}}\left\langle 0\left|\left[\alpha^{2}+\xi\left(\beta^{2}-\alpha^{2}\right)\right]+\right| 0\right\rangle_{a_{a}}\langle 1| \alpha \beta(1-2 \xi) \\
& \quad+|1\rangle_{a_{a}}\langle 0|\alpha \beta(1-2 \xi)+| 1\rangle_{a_{a}}\langle 1|\left[\beta^{2}+\xi\left(\alpha^{2}-\beta^{2}\right)\right] .
\end{aligned}
$$

We determine the optimum value of the parameter $\xi$ from the assumption that the distance (norm) between the two-mode density operators $\hat{\rho}_{a b}^{(o u t)}$ and $\hat{\rho}_{a b}^{(i d)}=\hat{\rho}_{a}^{(i d)} \otimes \hat{\rho}_{b}^{(i d)}$ is input-state independent. That is, we solve the equation

$$
\frac{\partial}{\partial \alpha^{2}} D_{a b}^{(2)}=0,
$$

where $D_{a b}^{(2)}=\operatorname{Tr}\left[\hat{\rho}_{a b}^{(o u t)}-\hat{\rho}_{a b}^{(i d)}\right]^{2}$ and the density operator $\hat{\rho}_{a b}^{(i d)}$ is given by Eq.(2.19b). The norm $D_{a b}^{(2)}$ in this case can be expressed as

$D_{a b}^{(2)}=\left(U_{11}\right)^{2}+2\left(U_{12}\right)^{2}+2\left(U_{13}\right)^{2}+\left(U_{22}\right)^{2}+2\left(U_{23}\right)^{2}+\left(U_{33}\right)^{2}$,

with the elements $U_{i j}$ given by the relations

$$
\begin{gathered}
U_{11}=\alpha^{4}-\alpha^{2}(1-2 \xi) ; \quad U_{12}=\sqrt{2} \alpha \beta\left[\alpha^{2}-\frac{1}{2}(1-2 \xi)\right] ; \\
U_{13}=\alpha^{2} \beta^{2} ; \quad U_{22}=2 \alpha^{2} \beta^{2}-2 \xi ; \quad \\
U_{23}=\sqrt{2} \alpha \beta\left[\beta^{2}-\frac{1}{2}(1-2 \xi)\right] ; \quad U_{33}=\beta^{4}-\beta^{2}(1-2 \xi) .
\end{gathered}
$$

Now the equation (3.13) can be solved with respect to the parameter $\xi$, for which we find $\xi=1 / 6$. For this value of $\xi$ the norm $D_{a b}^{(2)}$ is $\alpha^{2}$-independent and its value is equal to $2 / 9$.

\section{A. Some properties of the UQCM}

(1) Firstly we point out that the density operator $\hat{\rho}_{a}^{(i d)}$ is diagonal in the basis

$$
\left|\Phi_{1}\right\rangle_{a}=\alpha|0\rangle_{a}+\beta|1\rangle_{a} ; \quad\left|\Phi_{2}\right\rangle_{a}=\beta|0\rangle_{a}-\alpha|1\rangle_{a} .
$$

In this basis we have $\hat{\rho}_{a}^{(i d)}=\left|\Phi_{1}\right\rangle_{a}{ }_{a}\left\langle\Phi_{1}\right|$. In this same basis the density operator $\hat{\rho}_{a}^{(\text {out })}$ reads

$$
\hat{\rho}_{a}^{(\text {out })}=\frac{5}{6}\left|\Phi_{1}\right\rangle_{a_{a}{ }}\left\langle\Phi_{1}\left|+\frac{1}{6}\right| \Phi_{2}\right\rangle_{a_{a}}\left\langle\Phi_{2}\right|,
$$

from which it directly follows that the von Neumann entropy of the mode $a$ at the output of the copying machine is

$$
S_{a}=-k_{\mathrm{B}}\left[\frac{5}{6} \ln \left(\frac{5}{6}\right)+\frac{1}{6} \ln \left(\frac{1}{6}\right)\right] .
$$

Analogously we can evaluate the von Neumann entropy of the $a b$ subsystem (or, which is the same, the entropy of the QCM after the copying process) to be

$$
S_{a b}=S_{x}=-k_{\mathrm{B}}\left[\frac{1}{3} \ln \left(\frac{1}{3}\right)+\frac{2}{3} \ln \left(\frac{2}{3}\right)\right] .
$$

We see that both the von Neumann entropy of each output mode $a$ and $b$ separately, as well as the entropy of the two-mode subsystem $a b$ do not depend on the input pure state of the original mode $a$. Moreover, from the fact that the entropies under consideration fulfill the relation

$$
S_{a b}<S_{a}+S_{b}
$$

it follows that there does not exist a basis in which the density $\hat{\rho}_{a b}^{(\text {out })}$ can be represented in a factorized form $\hat{\rho}_{a}^{(\text {out })} \otimes \hat{\rho}_{b}^{(\text {out })}$. As we will see later this entanglement between the two output modes significantly affects the measurement procedure of the two modes after the copying. To be more specific, any measurement performed on mode $b$ affects the state of the mode $a$.

(2) Once we have found the basis in which both density operators $\hat{\rho}_{a}^{(i d)}$ and $\hat{\rho}_{a}^{(o u t)}$ are diagonal we can easily find the value of the fidelity parameter $F_{a}$ as introduced by Schumacher[4]. The fidelity parameter which we are interested in is given by Eq.(2.14) with $\hat{\rho}_{1}=\hat{\rho}_{a}^{(i d)}$ and $\hat{\rho}_{2}=\hat{\rho}_{a}^{(\text {out })}$. In our case the fidelity is equal to a constant value $\sqrt{5 / 6}$ for all input states states. We can conclude that the UQCM has that universal property to be the input state independent, that is all pure states are copied equally well.

(3) It is natural to ask how the copying machine under consideration will copy an input state described by the statistical mixture

$\hat{\rho}_{a}^{(i d)}=A|0\rangle_{a_{a}}\langle 0|+B| 0\rangle_{a_{a}}\langle 1|+B| 1\rangle_{a_{a}}\langle 0|+(1-A)| 1\rangle_{a}\langle 1|$,

where we assume for simplicity that $B$ is real. We note that from the condition $\operatorname{Tr}\left(\hat{\rho}_{a}^{(i d)}\right)^{2} \leq 1$ it follows that

$$
(1-2 A)^{2}+4 B^{2} \leq 1 .
$$

We find the Hilbert-Schmidt norm $D_{a}$ of the difference between the input state (3.19) and the corresponding output to be

$$
D_{a}=2 \xi^{2}(1-2 A)^{2}+2 B^{2}(1-\eta)^{2} .
$$

If we assume that $\eta=1-2 \xi$, then the norm (3.21a) reads

$$
D_{a}=2 \xi^{2}\left[(1-2 A)^{2}+4 B^{2}\right] \leq 2 \xi^{2},
$$

which means that the UQCM discussed here copies mixture states better than pure superposition states with the same diagonal density matrix elements.

If we assume $\xi=0$ [in this case the UQCM is identical to the WZQCM] then the statistical mixtures (3.19) such 
that $B=0$ are copied perfectly in the sense that the distance $D_{a}$ given by Eq. (3.21b) is equal to zero. Nevertheless one has to be aware of the fact that the two output modes are still strongly entangled which is reflected in the fact that the norm $D_{a b}^{(2)}$ has a value:

$$
D_{a b}^{(2)}=\operatorname{Tr}\left[\hat{\rho}_{a b}^{(o u t)}-\hat{\rho}_{a b}^{(i d)}\right]^{2}=4 A^{2}(1-A)^{2} .
$$

(4) We can compare the performance of the WoottersZurek QCM and the UQCM as discussed above if we compare the averaged values of the norms $D_{a}$ and $D_{a b}$ for the WZ QCM, which read respectively

$$
\bar{D}_{a}=1 / 3 ; \quad \bar{D}_{a b}=2 / 3
$$

with the input-state independent values of these parameters for the UQCM. We see that in the case of the UQCM the norm $D_{a}$ is 6 times smaller (on average) while $D_{a b}$ is 3 times smaller compared to $\bar{D}$ and $\bar{D}_{a b}$, respectively. These relations simply reflect the "high-quality" performance of the UQCM. It is still an open question whether the UQCM is the best (on average) QCM quantum mechanics would allow.

(5) The UQCM has that interesting property that the mean values of the operators $\hat{\sigma}_{x}$ and $\hat{\sigma}_{z}$ are scaled by copying. It can be found that irrespective of whether the input mode is in a pure state or a statistical mixture the following relations hold

$$
\left\langle\hat{\sigma}_{j}\right\rangle^{(\text {out })}=(1-2 \xi)\left\langle\hat{\sigma}_{j}\right\rangle^{(\text {in })} ; \quad j=x, z,
$$

where the relation $\eta=1-2 \xi$ has been taken into account. Obviously, for $\xi=1 / 6$ both $\left\langle\hat{\sigma}_{z}\right\rangle^{(\text {out })}$ and $\left\langle\hat{\sigma}_{x}\right\rangle^{(\text {out })}$ are scaled by the factor $2 / 3$. This is in contrast to the WZ QCM, when the mean value of the operator $\hat{\sigma}_{z}$ is preserved in the copying process, while $\left\langle\hat{\sigma}_{x}\right\rangle^{(\text {out })}=0$ irrespective of the input state. We note that the relations between the input and output mean values can be taken as definitions of particular copying machines. To be specific, one can associate the copying process with a given observation level, i.e. a set of observables, and impose particular conditions on input and output values of the observables associated with the given observation level. The relations between the input and output mean values then can be solved with respect to those parameters which specify the copying machine itself, i.e. the values ${ }_{x}\left\langle Q_{k l} \mid Q_{m n}\right\rangle_{x}$.

(6) The four state vectors $\left|Q_{0}\right\rangle_{x},\left|Q_{1}\right\rangle_{x},\left|Y_{0}\right\rangle_{x}$, and $\left|Y_{1}\right\rangle_{x}$ in terms of which the QCM transformation (3.2) is defined are not orthonormal. Using the GramSchmidt procedure one can defined a set of four orthonormal quantum-copying-machine basis states $\left|\bar{Q}_{0}\right\rangle_{x},\left|\bar{Q}_{1}\right\rangle_{x}$, $\left|\bar{Y}_{0}\right\rangle_{x}$, and $\left|\bar{Y}_{1}\right\rangle_{x}$. If we assume the relations (3.3), (3.4) and (3.8) defining the QCM under consideration and the relation $\eta=1-2 \xi$, the orthonormal states read:

$$
\left|\bar{Q}_{0}\right\rangle_{x}=\frac{\left|Q_{0}\right\rangle_{x}}{\sqrt{1-2 \xi}} ; \quad\left|\bar{Q}_{1}\right\rangle_{x}=\frac{\left|Q_{1}\right\rangle_{x}}{\sqrt{1-2 \xi}} ;
$$

$$
\left|\bar{Y}_{0}\right\rangle_{x}=\frac{2\left|Y_{0}\right\rangle_{x}-\left|Q_{1}\right\rangle_{x}}{\sqrt{6 \xi-1}} ; \quad\left|\bar{Y}_{1}\right\rangle_{x}=\frac{2\left|Y_{1}\right\rangle_{x}-\left|Q_{0}\right\rangle_{x}}{\sqrt{6 \xi-1}}
$$

from which it follows that one has to treat carefully the case of $\xi=1 / 6$, i.e. when the norm $D_{a b}$ is the inputstate independent. To be more specific, under the conditions given by Eqs.(3.3), (3.4) and (3.8) imposed on the copying-machine vectors $\left|Q_{i}\right\rangle_{x}$ and $\left|Y_{i}\right\rangle_{x}(i=0,1)$ with $\eta=2 / 3$ and $\xi=1 / 6$ we find the following relations

$$
\begin{gathered}
{ }_{x}\left\langle Q_{i} \mid Q_{i}\right\rangle_{x}=2 / 3 ; \quad{ }_{x}\left\langle Y_{i} \mid Y_{i}\right\rangle_{x}=1 / 6 ; \quad i=0,1 \\
{ }_{x}\left\langle Y_{1} \mid Y_{0}\right\rangle_{x}={ }_{x}\left\langle Q_{1} \mid Q_{0}\right\rangle_{x}=0 ; \\
{ }_{x}\left\langle Y_{0} \mid Q_{1}\right\rangle_{x}={ }_{x}\left\langle Y_{1} \mid Q_{0}\right\rangle_{x}=1 / 3,
\end{gathered}
$$

which means that the four copying-machine vectors are not linearly independent. They in fact lie in a 2dimensional sub-space of the original 4-dimensional space of the copying machine. In this 2-dimensional sub-space the copying-machine vectors have the following components:

$$
\begin{array}{r}
\left|Y_{0}\right\rangle_{x}=(1 / \sqrt{6}, 0) ; \quad\left|Y_{1}\right\rangle_{x}=(0,1 / \sqrt{6}) \\
\left|Q_{0}\right\rangle_{x}=(0, \sqrt{2 / 3}) ; \quad\left|Q_{1}\right\rangle_{x}=(\sqrt{2 / 3}, 0) .
\end{array}
$$

We see that the vectors $\left|Y_{i}\right\rangle_{x}$ can be expressed in terms of the vectors $\left|Q_{i}\right\rangle_{x}$ :

$$
\left|Y_{0}\right\rangle_{x}=\frac{1}{2}\left|Q_{1}\right\rangle_{x} \quad\left|Y_{1}\right\rangle_{x}=\frac{1}{2}\left|Q_{0}\right\rangle_{x}
$$

If we introduce two orthonormal basis states $|\uparrow\rangle$ and $|\downarrow\rangle$ in the two-dimensional state space, then we can express the copying-machine states $\left|Q_{0}\right\rangle_{x}$ in this basis as

$$
\left|Q_{0}\right\rangle_{x}=\sqrt{\frac{2}{3}}|\uparrow\rangle ; \quad\left|Q_{1}\right\rangle_{x}=\sqrt{\frac{2}{3}}|\downarrow\rangle .
$$

Consequently, the UQCM transformation (3.2) now reads

$$
\begin{aligned}
& |0\rangle_{a}|Q\rangle_{x} \longrightarrow \sqrt{\frac{2}{3}}|00\rangle|\uparrow\rangle+\sqrt{\frac{1}{3}}|+\rangle|\downarrow\rangle ; \\
& |1\rangle_{a}|Q\rangle_{x} \longrightarrow \sqrt{\frac{2}{3}}|11\rangle|\downarrow\rangle+\sqrt{\frac{1}{3}}|+\rangle|\uparrow\rangle,
\end{aligned}
$$

where the initial copying-machine state $|Q\rangle_{x}$ can be expressed as a linear superposition of the two basis states $|\uparrow\rangle$ and $|\downarrow\rangle$.

\section{MEASUREMENT OF THE ORIGINAL AND THE COPY STATE AT THE OUTPUT OF QCM}

From our previous discussion it follows that the original and the copy states (described by density operators 
$\hat{\rho}_{a}^{(\text {out })}$ and $\hat{\rho}_{b}^{(\text {out })}$, respectively) are highly entangled (see discussion in Section III). This means that any measurement performed on the mode $b$ will significantly affect the state of the mode $a$. This could defeat the purpose of a quantum copying machine because by measuring the copy we distort the original. Ideally we would like to have the copy and original independent so that if one is measured the other is undisturbed and available for future processing. We need to determine how close the copying machine of the previous section comes to this ideal.

What we shall do is to consider the effect of an unconditioned measurement of the $b$ mode on the state of the $a$ mode. Define the $b$ mode vector

$$
|s\rangle_{b}=u|0\rangle_{b}+v|1\rangle_{b} ; \quad|u|^{2}+|v|^{2}=1,
$$

and the corresponding projection operator $\hat{P}_{|s\rangle_{b}}=$ $|s\rangle_{b}\langle s|$. We start with an ensemble of copies and originals which has been produced by the copying machine and is described by the density matrix $\hat{\rho}_{a b}^{(\text {out })}$. We now measure $\hat{P}_{|s\rangle_{b}}$ for each element of the ensemble and, irrespective of the result, keep the resulting two-mode state. This results in the new ensemble

$$
\hat{\rho}_{a b}^{(\text {meas })}=\hat{P}_{|s\rangle_{b}} \hat{\rho}_{a b}^{(\text {out })} \hat{P}_{|s\rangle_{b}}+\hat{Q}_{|s\rangle_{b}} \hat{\rho}_{a b}^{(\text {out })} \hat{Q}_{|s\rangle_{b}},
$$

where $\hat{Q}_{|s\rangle_{b}}=\hat{I}_{b}-\hat{P}_{|s\rangle_{b}}$ and $\hat{I}_{b}$ is the $b$ mode identity operator. From this we obtain the $a$ mode reduced density matrix

$$
\hat{\rho}_{a}^{(\text {meas })}=\operatorname{Tr}_{b}\left(\hat{\rho}_{a b}^{(\text {meas })}\right) .
$$

The measurement of $\hat{P}_{|s\rangle_{b}}$ can yield either 0 or 1 . The probability of obtaining 1 is given by

$$
\operatorname{Tr}\left(\hat{P}_{|s\rangle_{b}} \hat{\rho}_{a b}^{(\text {out })} \hat{P}_{|s\rangle_{b}}\right)=\frac{1}{6}+\frac{2}{3}\left|\alpha u^{*}+\beta v^{*}\right|^{2} .
$$

It is clear from this equation that measurement of this probability will give us information about $\alpha$ and $\beta$. Thus by measuring the $b$ mode we do gain information about the quantum state of the input mode of the copying machine.

Now let us see what the effect of the $b$-mode measurement is on the $a$ mode. We note that

$\hat{\rho}_{a}^{(\text {out })}=\operatorname{Tr}_{b}\left[\left(\hat{P}_{|s\rangle_{b}}+\hat{Q}_{|s\rangle_{b}}\right) \hat{\rho}_{a b}^{(\text {out })}\left(\hat{P}_{|s\rangle_{b}}+\hat{Q}_{|s\rangle_{b}}\right)\right]=\hat{\rho}_{a}^{(\text {meas })}$

so that the $a$-mode density matrix after the unconditional measurement is the same as that before it. This result does not depend on $u$ and $v$ so we can choose to measure any projection in the $b$ mode.

Even though $\hat{\rho}_{a}^{(\text {out })}$ and $\hat{\rho}_{a}^{(i d)}$ are close $\left(D_{a}=1 / 18\right)$ they are not the same. However, because of the form of $\hat{\rho}_{a}^{(o u t)}$ it is possible to recover the expectation value of any operator in the state $\hat{\rho}_{a}^{(i d)}$ from it. In order to show this we express $\hat{\rho}_{a}^{(o u t)}$ as

$$
\hat{\rho}_{a}^{(o u t)}=\frac{2}{3} \hat{\rho}_{a}^{(i d)}+\frac{1}{6} \hat{I}_{a} .
$$

This implies that the density matrixes differ in a way that does not depend on $\alpha$ and $\beta$. Therefore, if $\hat{A}_{a}$ is an $a$-mode operator, then

$$
\operatorname{Tr}\left(\hat{A}_{a} \hat{\rho}_{a}^{(i d)}\right)=\frac{3}{2}\left[\operatorname{Tr}\left(\hat{A}_{a} \hat{\rho}_{a}^{(\text {out })}\right)-\frac{1}{6} \operatorname{Tr}\left(\hat{A}_{a}\right)\right],
$$

where $\operatorname{Tr}\left(\hat{A}_{a}\right)$ does not depend on $\alpha$ and $\beta$ and is, therefore, known.

In summary the output from the UQCM has the following property. If any projection is measured in the $b$ mode the unconditioned $a$-mode ensemble which results is close to the ideal output state, i. e. the input state, and can be used to find the expectation value of any $a$ mode operator in the ideal output state. In addition, the $b$-mode measurement provides us with information about the input state.

\section{COPYING STATES IN THE NEIGHBORHOOD OF GIVEN STATE}

Suppose that we want to build a copying machine which will copy to a high degree of fidelity, states in the neighborhood of a given quantum state. In order to get an idea of how to construct such a machine let us look at what would happen if the machine made perfect copies. We shall consider the input state given by Eq.(2.3) where $\beta$ is close to one and $\alpha$ is small in magnitude (in this section we consider $\alpha$ and $\beta$ to be complex numbers because this does matter to the results). If this state were copied perfectly we would have $\hat{\rho}_{a b}^{(i d)}$ given by Eq.(2.19b). Under the stated conditions on $\beta$ and $\alpha$ we have roughly that

$$
\alpha|0\rangle_{a}+\beta|1\rangle_{a} \longrightarrow \beta^{2}|11\rangle+\alpha(|10\rangle+|01\rangle) .
$$

This suggests that the copying machine specified by

$$
\begin{aligned}
|1\rangle_{a}|Q\rangle_{x} & \longrightarrow|1\rangle_{a}|1\rangle_{b}\left|Q_{1}\right\rangle_{x} \\
|0\rangle_{a}|Q\rangle_{x} & \longrightarrow \frac{1}{\sqrt{2}}\left(|1\rangle_{a}|0\rangle_{b}+|0\rangle_{a}|1\rangle_{b}\right)\left|Q_{1}\right\rangle_{x}
\end{aligned}
$$

where $|Q\rangle_{x}$ and $\left|Q_{1}\right\rangle_{x}$ are the initial and final states of the copying machine, respectively, would produce something like the desired action. Note that this machine is very different from the Wootters-Zurek machine in that while one basis vector is duplicated exactly, the other is completely changed. In fact, the state $|0\rangle_{a}$ is sent into a state which has no overlap at all with the perfectly cloned state $|0\rangle_{a}|0\rangle_{b}$.

We shall examine the action of this copying machine, but it is worthwhile to note at the beginning that there is a major problem with it. The factor of $1 / \sqrt{2}$, which is required by unitarity, means that we do not obtain the action indicated in Eq.(5.1). What we have is that

$$
\begin{gathered}
\left(\alpha|0\rangle_{a}+\beta|1\rangle_{a}\right)|Q\rangle_{x} \longrightarrow[\beta|11\rangle+\alpha|+\rangle]\left|Q_{1}\right\rangle_{x} \\
\equiv\left|\Psi_{1}\right\rangle_{a b}^{(\text {out })}\left|Q_{1}\right\rangle_{x} .
\end{gathered}
$$


We can determine how good job this copying machine does by looking at the difference between what it does and what it is supposed to do, i.e. we evaluate the Hilbert-Schmidt norm between the states described by the density operators $\hat{\rho}_{a b}^{(i d)}$ [see Eq.(2.12b)] and $\hat{\rho}_{a b}^{(\text {out })}$ [see Eq.(5.3)]. For the Hilbert-Schmidt norm $D_{a b}^{(2)}$ given by Eq.(2.15) we find the explicit expression

$$
D_{a b}^{(2)}=2-\left(\beta+\beta^{*}\right)\left(|\beta|^{2}+|\alpha|^{2} \sqrt{2}\right) .
$$

This can be simplified by expressing $\beta$ as $1-\delta \beta$ and using the normalization condition $|\beta|^{2}+|\alpha|^{2}=1$ to find the condition

$$
\delta \beta+\delta \beta^{*}=|\alpha|^{2}+|\delta \beta|^{2} .
$$

We then have that

$$
D_{a b}^{(2)}=(3-2 \sqrt{2})|\alpha|^{2}+|\delta \beta|^{2},
$$

where terms of order $|\alpha|^{4}$ and $|\delta \beta|^{2}|\alpha|^{2}$ have been dropped. Finally, we need to determine the size of $|\delta \beta|^{2}$. Setting $\delta \beta=r e^{i \theta}$ and substituting this into Eq.(5.5) we find that

$$
2 r \cos \theta-r^{2}=|\alpha|^{2} .
$$

This implies that unless $\theta$ is very close to $\pi / 2$, then $r$ will be of order $|\alpha|^{2}$. If $\delta \theta=\theta-\pi / 2$ is of order $|\alpha|$ or less, then $\mathrm{r}$ is of order $|\alpha|$. In either case, the right-hand side of Eq.(5.6) will be of order $|\alpha|^{2}$.

It is possible to do better than this in a certain sense. Consider the copying machine specified by

$$
\begin{aligned}
|1\rangle_{a}|Q\rangle_{x} & \longrightarrow \frac{1}{\sqrt{2}}\left(|11\rangle\left|Q_{1}\right\rangle_{x}+|00\rangle\left|Q_{0}\right\rangle_{x}\right) \\
|0\rangle_{a}|Q\rangle_{x} & \longrightarrow \frac{1}{\sqrt{2}}(|10\rangle+|01\rangle)\left|Q_{1}\right\rangle_{x},
\end{aligned}
$$

where ${ }_{x}\left\langle Q_{0} \mid Q_{0}\right\rangle_{x}={ }_{x}\left\langle Q_{1} \mid Q_{1}\right\rangle_{x}=1$ and ${ }_{x}\left\langle Q_{0} \mid Q_{1}\right\rangle_{x}=0$. With this copying machine we find that a superposition state goes into

$$
\begin{aligned}
\alpha|0\rangle_{a}+\beta|1\rangle_{a} \longrightarrow & \frac{1}{\sqrt{2}}[\beta|11\rangle+\alpha(|10\rangle+|01\rangle)]\left|Q_{1}\right\rangle_{x} \\
& +\frac{\beta}{\sqrt{2}}|00\rangle\left|Q_{0}\right\rangle_{x} .
\end{aligned}
$$

We define the vector in the first term on the right-hand side of Eq.(5.9) to be

$$
\left|\Psi_{2}\right\rangle_{a b}^{(o u t)}=\beta|11\rangle+\alpha(|10\rangle+|01\rangle) .
$$

This vector is much closer to the vector on the righthand side of Eq.(5.1) than is $\left|\Psi_{1}\right\rangle_{a b}^{(o u t)}$ given by Eq.(5.3). In fact, we find that the Hilbert-Schmidt norm between the density operator associated state vector (5.10) and the ideally copied state given by the density operator $(2.19 \mathrm{~b})$ is

$$
D_{a b}^{(2)}=|\delta \beta|^{2}+2|\alpha|^{2}|\delta \beta|^{2} .
$$

As long as $\theta$ is not too close to $\pi / 2$, the right-had side of this equation will be of order $|\alpha|^{4}$. This is a considerable improvement over what the Wootters-Zurek copying machine can do. There is, however, in this case, the problem of the term proportional to $|00\rangle$ in Eq.(5.9). What we can do with the output of this copying machine is to use it to calculate the expectation values of any operator which annihilates this state. That is, if $\hat{S}$ is an operator which has the property that $\hat{S}|00\rangle=0$, then we can get a very good estimate of the expectation value of $\hat{S}$ in the state $(2.19 \mathrm{~b})$ by calculating the expectation of $\hat{S}$ in the state on the right-hand side of Eq.(5.9) and multiplying the result by 2 . In this sense the copying machine specified by Eq.(5.8) does a good job of copying states in the neighborhood of $|1\rangle$.

Another possibility is to use a selective measurement to obtain the desired state from that in Eq. (5.9). If we measure the operator $\hat{P}_{00}=|00\rangle\langle 00|$ and obtain the value 0 the resulting two-mode density matrix is

$$
\hat{\rho}_{a b}^{(s e l)}=\frac{1}{1+|\alpha|^{2}}\left|\Psi_{2}\right\rangle_{a b}^{(\text {out })} \underset{a b}{(\text { out })}\left\langle\Psi_{2}\right| .
$$

This produces the desired result because $D_{a b}^{(2)}$ is again of the order of $|\alpha|^{4}$ as long as $\theta$ is not too close to $\pi / 2$. A nonselective measurement of any one-dimensional projection in the $b$ mode now gives us information about $\alpha$ and $\beta$ and leaves us with the reduced $a$-mode density matrix

$$
\hat{\rho}_{a}^{(s e l)}=\frac{1}{1+|\alpha|^{2}}\left(\hat{\rho}_{a}^{(i d)}+|\alpha|^{2}|1\rangle\langle 1|\right),
$$

which, for $|\alpha|^{2}<<1$, is close to $\hat{\rho}_{a}^{(i d)}$. Therefore, the transformation is Eq. (5.8) followed by a selective measurement gives us a good approximation to cloning for a limited range os states. The copy can be measured, providing information about the initial state, and the resulting $a$-mode density matrix is close to that of the input.

\section{CONCLUSIONS}

The Wootters-Zurek no-cloning theorem forbids the copying of an arbitrary quantum state. If one does not demand that the copy be perfect, however, possibilities emerge. We have examined a number of these. A quantum copying machine closely related to the one used by Wootters and Zurek in the proof of their no-cloning theorem copies some states perfectly and others poorly. That is, the quality of its output depends on the input. A second type of machine, which we called a universal quantum copying machine, has the property that the quality of its output is independent of its input. Finally, we examined a machine which combines a unitary transformation and a selective measurement to produce good copies of states in the neighborhood of a particular state. 
A problem with all of these machines is that the copy and original which appear at the output are entangled. This means that a measurement of one affects the other. We found, however, that a nonselective measurement of the one of the output modes will provide information about the input state and not disturb the reduced density matrix of the other mode. Therefore, the output of these xerox machines is useful.

There is further work to be done; we have only explored some of the possibilities. It would be interesting to know, for example, what the best input-state independent quantum copying machine is. One can also consider machines which make multiple copies. Does the quality of the copies decrease as their number increases? These questions remain for the future.

\section{Acknowledgements}

This work was supported by the National Science Foundation under grants INT 9221716 and PHY-9403601 and by the East-West Program of the Austrian Academy of Sciences under the contract No. 45.367/1-IV/6a/94 of the Österreichisches Bundesministerium für Wissenschaft und Forschung.

[1] W.K. Wootters and W.H. Zurek, Nature 299, 802 (1982).

[2] H. Barnum, C. Caves, C. Fuchs, R. Jozsa, and B. Schumacher, Los Alamos e-print archive, quant-ph/9511010, (1996).

[3] A.M. Perelomov: Generalized Coherent States and their Applications (Springer Verlag, Berlin, 1987).

[4] B. Schumacher, Phys. Rev. A 51, 2738 (1995).

[5] S.M. Barnett and S.J.D. Phoenix, Phys. Rev. A 40, 2404 (1989).

[6] V. Bužek, G. Adam, and G. Drobný, Ann. Phys. (NY) 245, 37 (1996), and references therein.

[7] A. Wehrl, Rev. Mod. Phys. 50, 221 (1978).

[8] H. Araki and E. Lieb, Commun. Math. Phys. 18, 160 (1970). 